\title{
MEASURABILITY OF NORM PROVED BY HAAR FUNCTIONS
}

\author{
H. C. FINLAYSON
}

ABSTRACT. A proof is given of the measurability of the supremum norm on Wiener space.

Classical Wiener measure is obtained by Gross [4] as a special case of his theorem on measurable norms on separable real Hilbert spaces. In this case the Hilbert space $C^{\prime}$ is the space of absolutely continuous functions on the unit interval which vanish at 0 and which have square integrable first derivative. The inner product of two such functions is the integral of the product of their derivatives and it is the supremum norm which is shown to be measurable. Gross uses his theorem on limits of measurable seminorms to prove this. However, since the definition of a measurable norm involves only the estimate of certain finite dimensional Lebesgue integrals, the short computational proof of this note may be of interest.

The completion $C$ of $C^{\prime}$ with the supremum norm is the space on which classical Wiener measure is defined. If $\left\{h_{i}(s)\right\}$ is the set of Haar functions normalized to be right continuous and to vanish at 1 , then

$$
\left\{B_{i}(t)=\int_{0}^{t} h_{i}(s) d s\right\}
$$

is chosen as an orthonormal basis of $C^{\prime}$. To say that $\left\{B_{i}(t)\right\}$ is a basis for $C^{\prime}$ means of course that

$$
\int_{0}^{1} B_{i}^{\prime}(t) B_{j}^{\prime}(t) d t=\delta_{i j}
$$

The reason for the Haar functions being useful may be that $\left\{B_{i}(t)\right\}$ is also a Schauder basis for C. In fact [3, p. 67] for any $x \in C$

$$
x(t)=\sum_{i=1}^{\infty} \int_{0}^{1} h_{i}(s) d x(s) B_{i}(t)
$$

in the uniform topology on $C$.

An indexing property of the Haar functions will be recalled. That is, there is a natural double indexing

$$
a_{0}^{(0)}(s), \quad a_{n}^{(k)}(s), \quad n=0,1,2, \ldots, k=1,2, \ldots, 2^{n} .
$$

Received by the editors May 21, 1974.

AMS (MOS) subject classifications (1970). Primary 28A40. 
Similarly the $B_{i}$ 's can be given a double indexing $\beta_{n}^{(k)}$. It will be convenient to speak of "the $n$th cycle of Haar functions" (or their integrals) by which is meant $\left\{\alpha_{n}^{(k)}: k=1,2, \ldots, 2^{n}\right\}$ (similar for $\beta^{\prime}$ s). Now if $P_{n}^{(k)}(x)$ is defined on $C^{\prime}$ by

$$
P_{n}^{(k)}(x)=\int_{0}^{1} x^{\prime}(s) \alpha_{n}^{(k)}(s) d s
$$

then from (1) it follows that for each $x \in C^{\prime}$

$$
x(t)=P_{0}^{(0)}(x) \beta_{0}^{(0)}(t)+\sum_{n=1}^{\infty} \sum_{k=1}^{2^{n}} P_{n}^{(k)}(x) \beta_{n}^{(k)}(t)
$$

in the uniform topology on $C$. Also, with the single indexing now used for the Haar functions, it is easy to see that to prove that the supremum norm is a measurable norm on $C^{\prime}$ it suffices to establish the following lemma. (An obvious slight modio fication of the proof shows that for any $\alpha \in(0,1 / 2)$ the Lip $\alpha$ norm on $C^{\prime}$ is measurable.)

Lemma. Let $\left\{B_{i}(t)\right\}$ be as above. For $\epsilon>0$ there exists $N>0$ such that

$\left[1 / \sqrt{2 \pi}^{l+1}\right] \int_{S}(l+1) \int \exp \left[-\left(x_{k}^{2}+\cdots+x_{k+l}^{2}\right) / 2\right] d x_{k} \cdots d x_{k+l}>1-\epsilon$ if $k>N$ where

$$
S=\left\{\left(x_{k}, \ldots, x_{k+l}\right): \sup _{t \in[0,1]}\left|\sum_{i=k}^{k+l} x_{i} B_{i}(t)\right|<\epsilon\right\} .
$$

Proof. If $B_{k}(t)$ and $B_{k+l}(t)$ are both in the $n$th cycle then

$$
\sup _{t \in[0,1]}\left|\sum_{i=k}^{k+l} x_{i} B_{i}(t)\right| \leq\left(1 / 2 \sqrt{2}^{n}\right) \max _{k \leq i \leq k+l}\left|x_{i}\right| .
$$

If $B_{k}(t)$ and $B_{k+l}(t)$ are in the $n$th and $(n+q)$ th cycle respectively then

$$
\sup _{t \in[0,1]}\left|\sum_{i=k}^{k+l} x_{i} B_{i}(t)\right| \leq \sum_{j=n}^{n+q} \max _{i \in j \text { th cycle }}\left|x_{i}\right| / 2 \sqrt{2}^{j} .
$$

Let $K=1 / 2 \sqrt[4]{2}(1-1 / \sqrt[4]{2})$ and restrict $\max _{i \in j \text { th cycle }}\left|x_{i}\right|$ by

$$
\max _{i \epsilon j \text { th cycle }}\left|x_{i}\right| / 2 \sqrt{2}^{j} \leq \epsilon / 2 \sqrt[4]{2}^{j} K
$$

i.e. $\max _{i \epsilon j \text { th cycle }}\left|x_{i}\right| \leq \sqrt[4]{2}^{j} \epsilon / K$. This restriction implies

$$
\sup _{t \in[0,1]}\left|\sum_{i=k}^{k+l} x_{i} B_{i}(t)\right| \leq \epsilon
$$

which defines the set over which the integral is to be estimated. Thus for $B_{k}(t)$ and $B_{k+l}(t)$ in the $n$th and $(n+q)$ th cycle respectively the integral is greater than 


$$
\begin{gathered}
\prod_{p=0}^{q}\left[2 \int_{0}^{\sqrt[4]{2^{n+p}} \epsilon / K} \exp \left(-u^{2} / 2\right) d u / \sqrt{2 \pi}\right]^{2^{n+p}} \\
=\prod_{p=0}^{q}\left\{1-2\left[1-\Phi\left(\sqrt[4]{2}^{n+p} \epsilon / K\right)\right]\right\}^{2^{n+p}} .
\end{gathered}
$$

( $\Phi$ is the normal distribution function, cf. e.g. [2].) This product is greater than $\Pi_{p=0}^{q}\left(1-a_{n+p}\right)^{2^{n+p}}$ where

$$
a_{j}=2 K \exp \left(-\sqrt{2}^{j} \epsilon^{2} / 2 K^{2}\right) /\left(\sqrt{2 \pi} \epsilon \sqrt[4]{2^{j}}\right) .
$$

But it is clear that $\sum_{j=0}^{\infty} 2^{j} a_{j}<\infty$ so that $\prod_{j=0}^{\infty}\left(1-a_{j}\right)^{2^{j}}$ converges and thus

$$
\prod_{p=0}^{q}\left(1-a_{n+p}\right)^{2^{n+p}}>1-\epsilon
$$

for all sufficiently large $n$. The proof is complete.

It does not seem easy to establish the convergence of the infinite product if, for instance, the sines, cosines of Legendre polynomials are used in place of the Haar functions.

Ciesielski [1] has also used the Haar functions in connection with Wiener type measures.

\section{REFERENCES}

1. Z. Ciesielski, Hölder conditions for realizations of Gaussian processes, Trans. Amer. Math. Soc. 99 (1961), 403-413. MR 24 \#A2431.

2. W. Feller, An introduction to probability theory and its applications. Vol. 1, Wiley, New York, 1950. MR 12, 424.

3. H. C. Finlayson, Approximation of Wiener integrals of functionals continuous in the uniform topology, Pacific J. Math. 34 (1970), 61-71.

4. L. Gross, Measurable functions on Hilbert space, Trans. Amer. Math. Soc. 105 (1962), 372-390. MR 26 \#5121.

DEPARTMENT OF MATHEMATICS AND ASTRONOMY, UNIVERSITY OF MANITOBA, WINNIPEG, MANITOBA, CANADA R3T 2N2 